\title{
CDK4/6 Inhibitors in the Treatment of Metastatic Breast Cancer
}

\author{
Chair: Johannes Ettl $\quad$ Ja \\ Participants: Jens Huober ${ }^{b}$ Diana Lüftner ${ }^{c} \quad$ Frederik Marméd $^{d}$ Rachel Würstlein $^{\mathrm{e}}$ \\ a Frauenklinik, Technische Universität München, Klinikum rechts der Isar, Munich, Germany; \\ ${ }^{\mathrm{b}}$ Brustzentrum, Frauenklinik, Universitätsklinikum UIm, UIm, Germany; \\ ${ }^{\mathrm{c}}$ Medizinische Klinik mit Schwerpunkt Hämatologie, Onkologie und Tumorimmunologie, \\ Charité Campus Benjamin Franklin, Berlin, Germany; \\ dUniversitäts-Frauenklinik, Nationales Centrum für Tumorerkrankungen, Heidelberg, Germany; \\ ${ }^{\mathrm{e}}$ Brustzentrum der Universität München, Standorte Großhadern und Maistraße - Innenstadt, Munich, Germany
}

With the marketing authorization of first-in-class CDK4/6 inhibitor palbociclib, a new type of orally available drug has been introduced into the therapy landscape of hormone receptor-positive $(\mathrm{HR}+)$ metastatic breast cancer $(\mathrm{MBC})$. Just recently, the Food and Drug Administration (FDA) approved another CDK4/6 inhibitor, ribociclib, in combination with an aromatase inhibitor (AI) for first-line treatment of postmenopausal women with estrogen receptor-positive (ER+) / human epidermal growth factor receptor 2-negative (HER2-) MBC. In numerous phase I-III trials, CDK4/6 inhibitors proved to be highly effective and remarkably well tolerated in combination with endocrine-based treatment for MBC.

The following expert discussion aims to shed light on new treatment strategies arising from the use of CDK4/6 inhibitors in clinical practice. 4 German experts in the field of $\mathrm{MBC}$ give their answers to 5 key questions concerning the present clinical relevance of CDK4/6 inhibitors.

\section{Question 1: What Is Your Favorite Clinical Setting in Which You Prefer to Combine Endocrine Therapy with CDK4/6 Inhibitors?}

Huober: CDK4/6 inhibitors are to be strongly considered in both the first-line setting combined with an AI (or fulvestrant if progressing while on an AI adjuvant) and the second-line setting with fulvestrant. In both settings, there was a clinically meaningful and significant improvement in progression-free survival (PFS), overall response rate (ORR), and clinical benefit rate (CBR). The toxicity profile of the combination therapy was predictable and manageable.

Lüftner: My suggestion in terms of patient selection is that all $\mathrm{HR}+$ patients who are not clearly in a high-risk situation should qualify for chemotherapy. This comprises a relative large spectrum of patients including those with visceral disease not at risk of organ failure or patients with bone involvement only.

Marmé: This is probably the first-line therapy in patients with a rather high disease burden and a strong need for remission. This may be the setting in which most can be gained from this combination: Many of these patients might previously have been regarded as candidates for chemotherapy and will have the benefit of avoiding chemotherapy for a long time; in addition, the expected gain in PFS is the largest in this setting. It is also important to control symptoms and avoid early disease progression in these cases. The combination of endocrine therapy and CDK4/6 inhibitors is ideal for accomplishing these goals. However, there are many other clinical settings in which these combinations are very good treatment options, so it is difficult to choose a favorite!

Würstlein: This combination is an option for any patient with HR+/HER2- MBC. Based on the data from the PALOMA 1-3 trials, we choose the combination with letrozole as first-line treatment in all patients not necessarily needing a chemo-induced ad hoc response. In further lines, treatment combination with fulvestrant is an option either within sequential endocrine treatment or after stabilization with chemotherapy and further progression.

\section{KARGER}

(c) 2017 S. Karger GmbH, Freiburg 


\section{Question 2: Which Group of ER+/HER2- MBC Patients Would You Recommend Not to Treat with CDK4/6 Inhibitors?}

Huober: Unfortunately, subgroup analyses of the Paloma and Monaleesa studies are not very helpful with regard to this question since there was no subgroup where the benefit of CDK4/6 inhibitors was not seen. Nevertheless, this does not automatically mean that every patient should have the combination treatment. We know that there are patients who do extremely well with endocrine treatment alone; however, there are currently no precise markers to reliably identify these patients. Moreover, we have no biomarkers that may indicate which patients are resistant to CDK4/6 inhibitors. These patients should be spared a more toxic combination therapy with CDK4/ inhibitors. I would discuss with patients the option of endocrine treatment only when there is limited asymptomatic metastatic disease (e.g., only very few bone lesions) which appears to be strongly endocrine-sensitive (long interval since completion of prior adjuvant endocrine treatment). Further, older age and significant comorbidities are also factors to support endocrine treatment alone.

Lüftner: I would in fact only exclude patients who cannot follow the observation measures necessary for safe treatment with CDK4/6 inhibitors. This could be patients who live a long distance from the institution or whose compliance is estimated to be low or uncertain.

Marmé: Patients who are truly endocrine-resistant but still relatively chemotherapy-naïve and in strong need of remission may not be treated with CDK4/6 inhibitors. In these cases, response rates for chemotherapy can be expected to be higher. With time progressing, many of these patients will have received a CDK4/6 inhibitor before getting into such a situation. Patients with very limited metastatic disease such as a single bone metastasis, which is relatively endocrine-naive and can be expected to be controlled with endocrine monotherapy, may not need the addition of a CDK4/6 inhibitor in first-line therapy. Another group of patients that I would not recommend to treat with CDK4/6 inhibitors are those who are unwilling or unable to comply with therapy management.

Würstlein: Good patient compliance/adherence is a prerequisite for use of oral palbociclib, and regular visits are necessary for discussion of blood results, potential dose modification, and followup. Patients with general neutropenia issues are not good candidates for oral palbociclib. Patients have to generally tolerate endocrine treatment with fulvestrant or an AI (so, for example, in further-line treatment, anticoagulation and fulvestrant may be contraindicated). Ineffective sequential endocrine treatment in $\mathrm{MBC}$ and repeated early progression may provide guidance in favor of chemotherapy. In first-line patients with singular bone metastasis, the use of a mono-AI or fulvestrant is an alternative option with the possibility of later adding palbociclib if needed.

\section{Question 3: From Your Personal Clinical Experience so far, How Do You Rate the Toxicity Profile of CDK4/6 Inhibitors?}

Huober: Mainly hematologic toxicity (decreased leukocytes and granulocytes) is seen, which is, however, manageable and does not usually bother the patient. Bothersome non-hematologic side effects with a negative impact on the patient's daily activities are rarely seen. However, the regular blood tests may be uncomfortable for some patients, which is something that would obviously not occur with endocrine therapy alone.

Lüftner: I call it 'management toxicity', meaning that the patients have to present to hospital every 2 weeks at the beginning. This means more traveling time compared to traditional endocrine treatment. Apart from that, I would say that the side effect profile is very favorable.

Marmé: From the patient's perspective, these agents are very well tolerated, with very little side effects affecting the patients' quality of life and well-being. Feedback from patients in these terms has been very favorable with hardly any complaints about tolerability. From the physician's perspective, the main toxicity, grade $3 / 4$ neutropenia, requires some attention. However, its management, including the timing of blood draws, dose interruptions, and possibly dose reductions, is well described and easily adopted. In this respect, therapy management is more elaborate compared to sending a patient off with a prescription for an AI and the request to return in 3 months' time. Nevertheless, the benefit for the patients outweighs these increased efforts.

Würstlein: In experienced centers, the toxicity management can be easily adopted. From the patient's point of view, the toxicity profile is well tolerated, as neutropenia (without infection/fever) can be managed without further problems by dose regulation.

\section{Question 4: Final Overall Survival Analyses of Phase II/II Trials Are Still Pending. Should These Analyses Fail to Show a Meaningful Overall Survival Advantage of CDK4/6 Inhibitors over Endocrine Monotherapy in MBC, How Would This Change Your Current View and Clinical Practice?}

Huober: Overall survival (OS) is an important parameter for patients with $\mathrm{MBC}$, albeit not the only one with clinical relevance. If disease can be controlled for a clinically meaningful longer time with only few additional side effects, which is the case with CDK4/6 inhibitors, I would not care too much about a missing OS benefit. In some patients for whom you would consider endocrine treatment alone, it may be an additional argument against the combination treatment.

Lüftner: Apart from 1 combination trial with fulvestrant and 
anastrozole, no endocrine therapy or biological modification of endocrine therapy has ever shown a survival benefit. It would not bother me. OS depends so much on post-trial treatments, and survival of $\mathrm{HR}+\mathrm{MBC}$ patients is so long that on OS benefit is extremely difficult to demonstrate. I would not insist on seeing a statistically significant survival benefit.

Marmé: It is important to remember that none of the trials have been powered to demonstrate an OS benefit. Therefore, failure to demonstrate such an OS advantage must not be interpreted as CDK4/6 inhibitors not providing an improvement in OS; it merely means that the OS benefit has not been demonstrated as might have been expected. Therefore, the influence of such a result on my current view will likely be marginal. In addition to the lack of statistical power, other factors, including the numerous lines of subsequent therapies, can impede the demonstration of an OS advantage. In the end, the data will have to be analyzed and interpreted once it is available.

Würstlein: PFS still is an objective in oncology, but expenses and efforts of patient management may play an important role in the choice of the optimal treatment irrespective of the OS effect.

\section{Question 5: In Your Opinion, What Are Promising Candidates for Tumor Biomarkers to Be Evaluated in Clinical Practice to Predict Response to CDK4/6 Inhibitors?}

Huober: None of the so far investigated tumor biomarkers have been successful in predicting response to CDK4/6 inhibitors. I do not currently see a promising biomarker to predict response to CDK4/6 inhibitors. There is still a lot of work to be done.

Lüftner: To my knowledge, all the interesting markers like PI3 kinase or CDK4/6 expression/mutation have been tested and proved to be non-predictive. I would like to see a predictive marker also for pharmacoeconomic reasons. However, if there is none and CDK4/6 inhibition is a general phenomenon, this would be fine as well.
Marmé: The obvious biomarkers, such as CDK4 and 6 as well as cyclin D1 amplification or expression of retinoblastoma protein (RB), have all been investigated without success so far. The same holds true for Ki67 and level of ER expression. The question of whether tissue from recent biopsies or circulating tumor DNA might be more suitable for identifying predictive biomarkers compared to archival tissue, is still open and warrants investigation. It might be easier to identify markers of resistance based on a strong biologic rationale, e.g. loss of CDK4/6 CNND1 or RB. For the moment, the most promising biomarker remains ER status. ESR1 mutations in circulating tumor DNA might help to select patients for fulvestrant rather than an $\mathrm{AI}$ as the combination partner.

Würstlein: So far, although multiple translational research has been performed, there is no biomarker. While we have to accept this, we should learn all we can from the trials performed as well as from clinical practice and real world data to establish an optimal treatment sequence and patient choice in $\mathrm{HR}+\mathrm{MBC}$.

\section{Participants}

Prof. Dr. Jens Huober

Brustzentrum, Frauenklinik

Universitätsklinikum Ulm

Prittwitzstr. 43, 89075 Ulm, Germany

Jens.Huober@uniklinik-ulm.de

PD Dr. Diana Lüftner

Universitätsmedizin Charité Mitte

Medizinische Klinik mit Schwerpunkt

Hämatologie, Onkologie und Tumorimmunologie

Charité Campus Benjamin Franklin

Hindenburgdamm 30, 12200 Berlin, Germany

diana.lueftner@charite.de

Dr. Frederik Marmé

Nationales Centrum für Tumorerkrankungen

Universitäts-Frauenklinik

Neuenheimer Feld 440, 69120 Heidelberg, Germany

frederik.marme@med.uni-heidelberg.de

Dr. med. Rachel Würstlein

Brustzentrum der Universität München

Standorte Großhadern und Maistraße - Innenstadt

Marchioninistr. 15, 81377 München, Germany

Rachel.Wuerstlein@med.uni-muenchen.de 